P448

ARE WE ENROLLING MEN WHO HAVE SEX WITH MEN WITH GREATEST HIV ACQUISITION RISK IN A PREEXPOSURE PROPHYLAXIS PROGRAM?

${ }^{1}$ Errol Fields*, ${ }^{2}$ Jessica Wagner, ${ }^{3}$ Christina Schumacher, ${ }^{3}$ Linxuan Wu, ${ }^{4}$ Aruna Chandran, ${ }^{5}$ Maisha Davis, ${ }^{6}$ Sarah Schmalzle, ${ }^{7}$ Joyce Jones, ${ }^{8}$ Jason Farley, ${ }^{1}$ Renata Sanders, ${ }^{9}$ Kathleen Page, ${ }^{10}$ Vicki Tepper, ${ }^{11}$ Adena Greenbaum, ${ }^{12}$ Jacky Jennings. ${ }^{1}$ Johns Hopkins University School of Medicine, Division of General Pediatrics and Adolescent Medicine, Department of Pediatrics, Baltimore, USA; ${ }^{2}$ Johns Hopkins University, Baltimore, USA; ${ }^{3}$ Johns Hopkins School of Medicine, Center for Child and Community Health Research, Baltimore, USA; ${ }^{4}$ Johns Hopkins University School of Medicine, Epidemiology, Baltimore, USA; ${ }^{5}$ Chase Brexton Health Care, Baltimore, USA; ${ }^{6}$ University of Maryland School of Medicine, Division of Infectious Diseases, Baltimore, USA; ${ }^{7}$ Johns Hopkins University School of Medicine, The I Bartlett Specialty Practice, Baltimore, USA; ${ }^{8}$ The Johns Hopkins University School of Nursing, Reach Initiative, Baltimore, USA; ${ }^{9}$ Baltimore City Health Department, Sexual Health/STD Clinics, Baltimore, USA; ${ }^{10}$ University of Maryland School of Medicine, Department of Pediatrics, Baltimore, USA; ${ }^{11}$ Baltimore City Health Department, Baltimore, USA; ${ }^{12}$ Johns Hopkins University School of Medicine, Center for Child and Community Health Research (CCHR), Baltimore, USA

10.1136/sextrans-2019-sti.532

Background Black men who have sex with men (BMSM) experience severe HIV disparities. Pre-exposure prophylaxis (PrEP) is a potential tool for reducing disparities especially when those at greatest risk are prioritized for PrEP delivery. To evaluate whether IMPACT, a multi-site PrEP demonstration project, enrolled MSM at greatest risk, this study examined associations between age, race, and HIV risk factors with PrEP program enrollment.

Methods Age, (13-24, 25+), race (non-Hispanic black, nonHispanic white, other), HIV risk factors (STI diagnosis, multiple partners in past 3 months and HIV-positive partner, condomless sex in past 12 months), and PrEP program enrollment (receipt AND acceptance of referral to PrEP provider) were collected from September 2015 through March 2018 for HIV-negative MSM screened by IMPACT. Multivariable logistic regression was used to determine the association between age, race, and HIV risk factors with PrEP program enrollment.

Results IMPACT screened 1883 MSM and enrolled 1413 (75.0\%). 1231(65.4\%) were non-Hispanic black; 484(25.7\%) were 13-24; 593(31.5\%) had recent STI; 802(43\%) reported multiple sex partners; $832(44 \%)$ reported condomless sex and $325(17.3 \%)$ reported an HIV-positive sex partner. Individuals with black race (compared to white) ( $\mathrm{AOR}=0.2 ; 95 \%$ $\mathrm{CI}=0.15-0.31)$, an HIV-positive sex partner $(\mathrm{AOR}=0.32 ; 95 \%$ $\mathrm{CI}=0.24-0.42)$, and a recent STI $(\mathrm{AOR}=0.30 ; 95 \% \mathrm{CI}=0.24$ 0.39 ) were significantly less likely to be enrolled. Individuals with multiple partners $(\mathrm{AOR}=1.34 ; 95 \% \mathrm{CI}=1.04-1.73)$, age 13-24 $(\mathrm{AOR}=1.99 ; 95 \% \mathrm{CI}=1.49-2.65)$, and condomless sex $(\mathrm{AOR}=2.24 ; 95 \% \quad \mathrm{CI}=1.74-2.88)$ were significantly more likely to be enrolled.

Conclusion These findings suggest significant barriers to PrEP enrollment for BMSM which may widen rather than reduce HIV disparities. Importantly, youth and MSM reporting condomless sex and multiple partners were more likely to be enrolled. Yet, negative associations between enrollment and black race, recent STI, HIV-positive sex partners suggest critical challenges engaging MSM at greatest risk for HIV which may diminish PrEP effectiveness as a public health prevention tool if unaddressed.

Disclosure No significant relationships.
ART, PrEP, gay bisexual and other men who have sex with men, USA

\section{P449 WHO'S GOT PREP? ASSOCIATION BETWEEN RACE, RECENT STI AND RECEIPT OF PREP PRESCRIPTION AMONG MSM ENROLLED IN A PREP PROGRAM}

${ }^{1}$ Errol Fields*, ${ }^{2}$ Jessica Wagner, ${ }^{3}$ Christina Schumacher, ${ }^{3}$ Linxuan Wu, ${ }^{4}$ Aruna Chandran, ${ }^{5}$ Maisha Davis, ${ }^{6}$ Sarah Schmalzle, ${ }^{7}$ Joyce Jones, ${ }^{8}$ Jason Farley, ${ }^{1}$ Renata Sanders, ${ }^{9}$ Kathleen Page, ${ }^{10}$ Vicki Tepper, ${ }^{11}$ Adena Greenbaum, ${ }^{12}$ Jacky Jennings. ${ }^{1}$ Johns Hopkins University School of Medicine, Division of General Pediatrics and Adolescent Medicine, Department of Pediatrics, Baltimore, USA; ${ }^{2}$ Johns Hopkins University, Baltimore, USA; ${ }^{3}$ Johns Hopkins School of Medicine, Center for Child and Community Health Research, Baltimore, USA; ${ }^{4}$ Johns Hopkins University School of Medicine, Epidemiology, Baltimore, USA; ${ }^{5}$ Chase Brexton Health Care, Baltimore, USA; ${ }^{6}$ University of Maryland School of Medicine, Division of Infectious Diseases, Baltimore, USA; ${ }^{7}$ Johns Hopkins University School of Medicine, The JBartlett Specialty Practice, Baltimore, USA; ${ }^{8}$ The Johns Hopkins University School of Nursing, Reach Initiative, Baltimore, USA; ${ }^{9}$ Baltimore City Health Department, Sexual Health/std Clinics, Baltimore, USA; ${ }^{10}$ University of Maryland School of Medicine, Department of Pediatrics, Baltimore, USA; ${ }^{11}$ Baltimore City Health Department, Baltimore, USA; ${ }^{12}$ Johns Hopkins University School of Medicine, Center for Child and Community Health Research (CCHR), Baltimore, USA

\subsection{6/sextrans-2019-sti.533}

Background Pre-exposure prophylaxis (PrEP) is a potential tool for reducing racial HIV disparities by reducing HIV acquisition among those at greatest risk. A recent bacterial STI is an important biomarker of HIV acquisition risk and represents one target for prioritizing PrEP in populations with the greatest HIV burden. Receipt of a PrEP prescription is an important step of the PrEP cascade. This study assessed whether race and recent STI diagnoses were associated with receipt of a PrEP prescription among men who have sex with men (MSM) in a PrEP program.

Methods Race (non-Hispanic black, non-Hispanic white, other), recent STI diagnosis (self-reported diagnosis in past 3 months) and data on receipt of a PrEP prescription among MSM who met clinical eligibility criteria for PrEP were collected from September 2015 through March 2018. Participants were enrolled at one of six clinical sites participating in IMPACT, a multi-site PrEP demonstration project. Multivariable logistic regression analysis was used to determine the associations between race and recent STI diagnosis with receipt of a PrEP prescription.

Results IMPACT screened 308 MSM as clinically eligible for PrEP and 287(93.2\%) received a PrEP prescription; 153 (49.7\%) were non-Hispanic black and 109(35.4\%) reported an STI diagnosis. MSM with black race (compared to white) $(\mathrm{AOR}=0.170 ; 95 \% \mathrm{CI}=0.047-0.607)$ were significantly less likely to receive a PrEP prescription. MSM with a recent STI diagnosis $(\mathrm{AOR}=4.874 ; 95 \% \mathrm{CI}=1.377-17.249)$ were more likely to receive a PrEP prescription.

Conclusion MSM with recent STI diagnosis were more likely to receive PrEP prescriptions. The finding that black MSM are less likely to receive prescriptions suggest a barrier to PrEP delivery that may widen rather than reduce HIV disparities. Identifying and understanding these barriers to PrEP delivery are an important target for future research. Disclosure No significant relationships. 